\title{
Stability Analysis of Large-Scale Distributed Networked Control Systems with Random Communication Delays: A Switched System Approach
}

\author{
Kooktae Lee and Raktim Bhattacharya ${ }^{a}$ \\ ${ }^{a}$ Kooktae Lee and Raktim Bhattacharya are with the Department of Aerospace \\ Engineering, Texas A\&M University, College Station, TX 77843-3141, USA, \\ \{animodor, raktim\}@tamu.edu.
}

\begin{abstract}
In this paper, we consider the stability analysis of large-scale distributed networked control systems with random communication delays. The stability analysis is performed in the switched system framework, particularly as the Markov jump linear system. There have been considerable research on stability analysis of the Markov jump systems. However, these methods are not applicable to large-scale systems because large numbers of subsystems result in extremely large number of switching modes. To circumvent this scalability issue, we propose a new reduced mode model for stability analysis, which is computationally scalable. We also consider the case in which the transition probabilities for the Markov jump process contain uncertainties. We provide a new method that estimates bounds for uncertain Markov transition probability matrix to guarantee the system stability. Numerical example verifies the computational efficiency of the proposed methods.
\end{abstract}

Keywords: Large-scale distributed networked control system, Markov jump linear system, switched system, random communication delays

\section{Introduction}

A networked control system (NCS) is a system that is controlled over a communication network. Recently, NCSs have attracted considerable research interest due to emerging networked control applications. For example, NCSs are broadly used in applications including traffic monitoring, networked autonomous mobile agents, chemical plants, sensor networks, and distributed 
software systems in cloud computing architectures. Due to the communication network, communication delays or communication losses may occur, resulting in performance degradation or even instability. Therefore, it has led various researchers to analyze NCSs associated with communication delays [1], [2], [3], [4], [5], [6], [7], [8]. Particularly in [6], the NCS with communication delays was analyzed by adopting the switched system [7], [8], [9], [10], [11], which refers to the dynamical system consists of a family of subsystems and a switching logic governing switching between subsystems.

In this paper, we study large-scale distributed networked control system (DNCS), which denotes NCS with a large number of spatially distributed subsystems (or agents). For such large-scale systems, our primary goal is to analyze system stability when random communication delays exist. Typically, the system behavior with random communication delays have been widely modeled as Markov jump linear system (MJLS) [6], [12], [13], [14], [15], [16] where the switching sequence is governed by a Markovian process. Since stability has been one of the major concerns, considerable effort has been on stability analysis of MJLS [17], [10], [18], [19], [20]. However, these results are only applicable to the systems with a small number of switching modes. For large-scale DNCSs, in which we are particularly interested, give rise to an extremely large number of switching modes. Thus, previous conditions developed for the stability analysis of MJLSs cannot be evaluated for large-scale DNCSs as they are not computationally tractable. Although the literature [21] recently investigated the switched system that circumvents computation issues associated with a large number of switching modes, it is developed for independent and identically distributed (i.i.d.) switching. We consider Markovian switching in this paper. In addition, we are also interested in large-scale DNCSs where the transition probabilities are inaccurately known[20], [22], [23]. This can happen because in practice it is difficult to accurately estimate the Markov transition probability matrix that models the random communication delays.

This paper provides two key contributions to analyze the stability of largescale DNCSs with random communication delays. Firstly, we guarantee the mean square stability of such systems by introducing a reduced mode model. We prove that the mean square stability for individual switched system implies a necessary and sufficient stability condition for the entire DNCS. This drastically reduces the number of modes necessary for analysis. Secondly, we present a new method to estimate the bound for uncertain Markov transition probability matrix, in order to guarantee the system stability. These results 
enable us to analyze large-scale systems in a computationally tractable manner.

Rest of this paper is organized as follows. We introduce the problem for the large-scale DNCS in section 2. Section 3 presents the switched system framework for the stability analysis with communication delays. In Section 4, we propose the reduced mode model to efficiently analyze stability. Section 5 quantifies the stability region and bounds for uncertain Markov transition probability matrix. This is followed by the application of the proposed method to an example system in section 6 , and we conclude the paper with section 7 .

Notation: The set of real numbers is denoted by $\mathbb{R}$. The symbols $\|\cdot\|$ and $\|\cdot\|_{\infty}$ stand for the Euclidean and infinity norm, respectively. The symbol \# $(\cdot)$ denotes the cardinality - the total number of elements in the given set. In addition, the symbols $\operatorname{tr}(\cdot), \rho(\cdot), \otimes$, and $\operatorname{diag}(\cdot)$ represent trace operator, spectral radius, Kronecker product, and block diagonal matrix operator, respectively.

\section{Problem Formulation}

\subsection{Distributed networked control system with no delays}

Consider a discrete-time dynamics of each agent in the DNCS, given by:

$$
x_{i}(k+1)=\sum_{j \in \mathcal{N}_{i}} A_{i j} x_{j}(k), \quad i=1,2, \ldots, N
$$

where $k$ is a discrete-time index, $N$ is the total number of agents (subsystems), $x_{i} \in \mathbb{R}^{n}$ is a state for the $i^{t h}$ agent, $\mathcal{N}_{i}$ is a set of neighbors for $x_{i}$ including the agent $x_{i}$ itself, and $A_{i j} \in \mathbb{R}^{n \times n}$ is a time-invariant system matrix that represents the linear interconnections between agents. Note that we have $A_{i j}=0$ if there is no interconnection between the agents $i$ and $j$.

To represent the entire systems dynamics, we define the state $x(k) \in$ $\mathbb{R}^{N n \times N n}$ as $x(k) \triangleq\left[x_{1}(k)^{\top}, x_{2}(k)^{\top}, \ldots, x_{N}(k)^{\top}\right]^{\top}$. Then, the system dynamics of the DNCS is given as

$$
x(k+1)=\mathcal{A} x(k),
$$


with the following definition for the matrix $\mathcal{A} \in \mathbb{R}^{N n \times N n}$

$$
\begin{aligned}
& \mathcal{A} \triangleq\left[\begin{array}{ccccc}
A_{11} & A_{12} & A_{13} & \cdots & A_{1 N} \\
A_{21} & A_{22} & A_{23} & \cdots & A_{2 N} \\
A_{31} & A_{32} & A_{33} & \cdots & A_{3 N} \\
\vdots & \vdots & \vdots & \ddots & \vdots \\
A_{N 1} & A_{N 2} & A_{N 3} & \cdots & A_{N N}
\end{array}\right], \\
& A_{i j}=\left\{\begin{array}{l}
0, \text { if no connection between the agents } i \text { and } j, \\
A_{i j}, \text { otherwise. }
\end{array}\right.
\end{aligned}
$$

For the discrete-time system in (2), it is well known that the system is stable if and only if the condition $\rho(\mathcal{A})<1$ is satisfied. Throughout the paper we assume that the system without communication delays, defined in (2), is stable. Then, we address stability in the presence of random communication delays. We remind the reader that $N$ is very large.

\subsection{DNCS with communication delays}

Often, network communication between agents encounter time delays or packet losses while sending and receiving data. We denote the symbol $\tau$ as random communication delays and assume that $\tau$ has a discrete value bounded by $0 \leq \tau \leq \tau_{d}<\infty$, where $\tau_{d}$ is a finite-valued maximum delay. Then, the dynamics for the agent $i$ with communication delays can be expressed as:

$$
x_{i}(k+1)=\sum_{j \in \mathcal{N}_{i}} A_{i j} x_{j}\left(k^{*}\right), \quad i=1,2, \ldots, N
$$

where $k^{*} \triangleq k-\tau$. Note that we have no communication delays when $i=j$ because there is no communication in this case.

The random communication delay, represented by the term $k^{*}$, forms a stochastic process. To analyze the stability of the DNCS, we define an augmented state $X(k)$ as $X(k) \triangleq\left[x(k)^{\top}, x(k-1)^{\top}, \cdots, x\left(k-\tau_{d}\right)^{\top}\right]^{\top} \in$ $\mathbb{R}^{N n q \times N n q}$, where $q \triangleq \tau_{d}+1$. Then, the dynamics for the entire system is given by

$$
X(k+1)=W(k) X(k)
$$


where $W(k) \triangleq\left[\begin{array}{ccccc}\tilde{A}_{1}(k) & \tilde{A}_{2}(k) & \cdots & \tilde{A}_{q-1}(k) & \tilde{A}_{q}(k) \\ I & 0 & \cdots & 0 & 0 \\ 0 & I & \cdots & 0 & 0 \\ \vdots & \vdots & \ddots & \vdots & \vdots \\ 0 & 0 & \cdots & I & 0\end{array}\right] \in \mathbb{R}^{N n q \times N n q}$

the matrix $I$ denotes an identity matrix with proper dimensions, and the time-varying matrices $\tilde{A}_{j}(k) \in \mathbb{R}^{N n \times N n}, j=1,2, \ldots, q$, model the randomness in the communication delays between neighboring agents.

\section{Switched System Approach}

Without loss of generality, the dynamics of the large-scale DNCS with communication delays in (4) can be transformed into a switched system framework as follows:

$$
x(k+1)=W_{\sigma(k)} x(k), \quad \sigma(k) \in\{1,2, \cdots, m\},
$$

where $W_{\sigma(k)}$ is the time-invariant matrix, representing communication delays in agents, $\{\sigma(k)\}$ is the switching sequence, and $m$ is the total number of switching modes. When the switching sequence $\{\sigma(k)\}$ is stochastic, (5) is referred to as a stochastic switched linear system or a stochastic jump linear system, according to the literature [7]. For the stochastic switched linear system, the switching sequence $\{\sigma(k)\}$ is governed by the mode-occupation switching probability $\pi(k)=\left[\pi_{1}(k), \pi_{2}(k), \ldots, \pi_{m}(k)\right]$, where $\pi_{i}$ is a fraction number, satisfying $\sum_{i=1}^{m} \pi_{i}=1$ and $0 \leq \pi_{i} \leq 1, \forall i$. In this case, each $\pi_{i}$ denotes the modal probability corresponding to each mode dynamics $W_{i}$. In order to properly describe the behavior of random communication delays, it is necessary to adopt a certain switching logic, which is used to update the switching probability $\pi(k)$. For this purpose, the MJLS framework has been widely employed [12], [13], [14], [15], [16]. Thus, we make the following assumption in our analysis.

- Assumption: Consider the stochastic jump linear system (5) with the switching probability $\pi(k)=\left[\pi_{1}(k), \pi_{2}(k), \ldots, \pi_{m}(k)\right]$. Then, $\pi(k)$ is updated by the Markovian process given by $\pi(k+1)=\pi(k) P$, where $P \in \mathbb{R}^{m \times m}$ is the Markov transition probability matrix.

Since the MJLS is a family of the stochastic switched linear system, various stability notions can be defined [10]. In this paper, we will consider the mean square stability condition, defined below. 
Definition 3.1. (Definition 1.1 in [11]) The MJLS is said to be mean square stable if for any initial condition $x_{0}$ and arbitrary initial probability distribution $\pi(0), \lim _{k \rightarrow \infty} \mathbb{E}\left[\left\|x\left(k, x_{0}\right)\right\|^{2}\right]=0$.

The total number of switching modes $m$ depends on the size $q$ and $N$. Since the communication delays take place independently while receiving and sending the data for each agent, $m$ is calculated by counting all possible scenarios to distribute every matrices $A_{i j} \in \mathbb{R}^{n \times n}$ for $i \neq j$ in the block matrix $\mathcal{A} \in \mathbb{R}^{N n \times N n}$ given in (2), into each $\tilde{A}_{j}(k) \in \mathbb{R}^{N n \times N n}, j=1,2, \ldots, q$, given in (4), which results in $m=q^{N(N-1)}$. Note that the large-scale DNCS that has considerably large $N$ results in extremely large $m$, which makes current analysis tools useless as they are computationally intractable.

Before we further proceed, we introduce the following proposition that was developed for the stability analysis of the MJLS.

Proposition 3.1. (Theorem 1 in [18]) The MJLS with the Markov transition probability matrix $P$ is mean square stable if and only if

$$
\rho\left(\left(P^{\top} \otimes I\right) \operatorname{diag}\left(W_{j} \otimes W_{j}\right)\right)<1,
$$

where I is an identity matrix with a proper dimension,

$\operatorname{diag}\left(W_{j} \otimes W_{j}\right) \triangleq\left[\begin{array}{ccccc}\left(W_{1} \otimes W_{1}\right) & 0 & 0 & \cdots & 0 \\ 0 & \left(W_{2} \otimes W_{2}\right) & 0 & \cdots & 0 \\ \vdots & & \ddots & & \vdots \\ 0 & 0 & & \left(W_{m-1} \otimes W_{m-1}\right) & 0 \\ 0 & 0 & \ldots & 0 & \left(W_{m} \otimes W_{m}\right)\end{array}\right]$,

and $m$ is the total number of the switching modes.

For the given set of matrices $\left\{W_{\sigma(k)}\right\}_{\sigma(k)=1}^{m}$ and the transition probability matrix $P$, one can always compute the spectral radius given in (6), and hence guarantee the system stability.

Unfortunately, this condition is not applicable to large-scale DNCSs due to enormously large $m$. For example, even with $q=2$ and $N=100$, we have $m=2^{100 \times 99}$. It is not practically possible to compute the spectral radius in (6) for such problems. To circumvent this scalability issue, we present a new analysis approach for such large-scale DNCSs in the next section. 


\section{Stability with Reduced Mode Dynamics}

In this section, we define a new augmented state to reduce the mode numbers as follows:

$$
\hat{x}_{i}(k) \triangleq\left[\tilde{x}_{i}(k)^{\top}, \tilde{x}_{i}(k-1)^{\top}, \cdots, \tilde{x}_{i}\left(k-\tau_{d}\right)^{\top}\right]^{\top} \in \mathbb{R}^{\hat{n}_{i} n q},
$$

where $\hat{n}_{i} \triangleq \#\left(\mathcal{N}_{i}\right), \mathcal{N}_{i}$ stands for the set of neighbors to $x_{i}$ including itself, $\tilde{x}_{i}(k) \triangleq\left[x_{i}(k)^{\top}, x_{j}(k)^{\top}\right]^{\top} \in \mathbb{R}^{\hat{n}_{i} n}$, and $x_{j}(k) \in \mathbb{R}^{n}, j \in \mathcal{N}_{i}$, denotes all states that are neighbor to $x_{i}(k) \in \mathbb{R}^{n}$.

Then, we can construct a switched linear system framework similarly to (5) as follows:

$$
\hat{x}_{i}(k+1)=\hat{W}_{\sigma_{i}(k)}^{i} \hat{x}_{i}(k), \quad \sigma_{i}(k) \in\left\{1,2, \ldots, m_{i}\right\},
$$

where $\hat{W}_{\sigma_{i}(k)}^{i} \triangleq\left[\begin{array}{ccccc}\hat{A}_{1}(k) & \hat{A}_{2}(k) & \cdots & \hat{A}_{q-1}(k) & \hat{A}_{q}(k) \\ I & 0 & \cdots & 0 & 0 \\ 0 & I & \cdots & 0 & 0 \\ \vdots & \vdots & \ddots & \vdots & \vdots \\ 0 & 0 & \cdots & I & 0\end{array}\right] \in \mathbb{R}^{\hat{n}_{i} n q \times \hat{n}_{i} n q}$

with the time-varying matrix $\hat{A}_{j}(k) \in \mathbb{R}^{\hat{n}_{i} n \times \hat{n}_{i} n}, j=1,2, \ldots, q$. In this case, the total number of the switching modes for (7) is given by $m_{i}=q^{\hat{n}_{i}\left(\hat{n}_{i}-1\right)}$.

By implementing the reduced mode model given in (7), we provide a computationally efficient condition for stability analysis of the original DNCS in the following theorem.

Theorem 4.1. Consider the large-scale DNCS (5) with Markovian communication delays associated with the transition probability matrix $P$. The necessary and sufficient condition for the mean square stability of this system is given by

$$
\rho\left(\left(P^{i^{\top}} \otimes I\right) \operatorname{diag}\left(\hat{W}_{j}^{i} \otimes \hat{W}_{j}^{i}\right)\right)<1, \quad \forall i=1,2, \ldots, N,
$$

where $P^{i} \in \mathbb{R}^{m_{i} \times m_{i}}$ is the transition probability matrix for the reduced mode MJLS given in (7), I is an identity matrix with a proper dimension, $N$ is the total number of the agents in the system, $m_{i}=q^{\hat{n}_{i}\left(\hat{n}_{i}-1\right)}$ is the total mode 
numbers for the reduce mode MJLS, and

$$
\operatorname{diag}\left(\hat{W}_{j}^{i} \otimes \hat{W}_{j}^{i}\right) \triangleq\left[\begin{array}{ccccc}
\left(\hat{W}_{1}^{i} \otimes \hat{W}_{1}^{i}\right) & 0 & 0 & \ldots & 0 \\
0 & \left(\hat{W}_{2}^{i} \otimes \hat{W}_{2}^{i}\right) & 0 & \cdots & 0 \\
\vdots & & \ddots & & \vdots \\
0 & 0 & & \left(\hat{W}_{m_{i}-1}^{i} \otimes \hat{W}_{m_{i}-1}^{i}\right) & 0 \\
0 & 0 & \ldots & 0 & \left(\hat{W}_{m_{i}}^{i} \otimes \hat{W}_{m_{i}}^{i}\right)
\end{array}\right]
$$

Proof. Let the matrix $Q^{i}(k)$ be of the form $Q^{i}(k) \triangleq \mathbb{E}\left[\hat{x}_{i}(k) \hat{x}_{i}(k)^{\top}\right]$. Then, $Q^{i}(k)$ is alternatively obtained by the following equation: $Q^{i}(k)=\sum_{s=1}^{m_{i}} Q_{s}^{i}(k)$, where $Q_{s}^{i}(k) \triangleq \mathbb{E}\left[\hat{x}_{i}(k) \hat{x}_{i}(k)^{\top} \mid \sigma_{i}(k)=s\right] \pi_{s}^{i}(k)$, and $\pi_{s}^{i}(k) \triangleq \operatorname{Pr}\left(\sigma_{i}(k)=s\right)$. Then, $Q_{s}^{i}(k)$ satisfies

$$
\begin{aligned}
& Q_{s}^{i}(k)=\sum_{r=1}^{m_{i}} \mathbb{E}\left[\hat{x}_{i}(k) \hat{x}_{i}(k)^{\top} \mid \sigma_{i}(k)=s, \sigma_{i}(k-1)=r\right] \\
& \operatorname{Pr}\left(\sigma_{i}(k-1)=r \mid \sigma_{i}(k)=s\right) \pi_{s}^{i}(k) \\
& =\sum_{r=1}^{m_{i}} \mathbb{E}\left[\hat{x}_{i}(k) \hat{x}_{i}(k)^{\top} \mid \sigma_{i}(k)=s, \sigma_{i}(k-1)=r\right] \\
& \underbrace{\operatorname{Pr}\left(\sigma_{i}(k)=s \mid \sigma_{i}(k-1)=r\right)}_{\triangleq p_{r s}^{i}} \pi_{r}^{i}(k-1) \\
& =\sum_{r=1}^{m_{i}} p_{r s}^{i} \mathbb{E}\left[\hat{x}_{i}(k) \hat{x}_{i}(k)^{\top} \mid \sigma_{i}(k)=s, \sigma_{i}(k-1)=r\right] \pi_{r}^{i}(k-1) \\
& =\sum_{r=1}^{m_{i}} p_{r s}^{i} \mathbb{E}\left[\hat{W}_{\sigma_{i}(k-1)}^{i} \hat{x}_{i}(k-1) \hat{x}_{i}(k-1)^{\top} \hat{W}_{\sigma_{i}(k-1)}^{i^{\top}} \mid \sigma_{i}(k-1)=r\right] \pi_{r}^{i}(k-1) \\
& =\sum_{r=1}^{m_{i}} p_{r s}^{i} \hat{W}_{r}^{i} \underbrace{\mathbb{E}\left[\hat{x}_{i}(k-1) \hat{x}_{i}(k-1)^{\top} \mid \sigma_{i}(k-1)=r\right] \pi_{r}^{i}(k-1)}_{=Q_{r}^{i}(k-1)} \hat{W}_{r}^{i^{\top}} \\
& =\sum_{r=1}^{m_{i}} p_{r s}^{i} \hat{W}_{r}^{i} Q_{r}^{i}(k-1) \hat{W}_{r}^{i^{\top}}
\end{aligned}
$$

In the second equality of above equation, $p_{r s}^{i}$ denotes the mode transition probability from $r$ to $s$ in the Markov transition probability matrix $P^{i}$. 
Taking the vectorization in above equation results in

$$
\begin{aligned}
\operatorname{vec}\left(Q_{s}^{i}(k)\right) & =\operatorname{vec}\left(\sum_{r=1}^{m_{i}} p_{r s}^{i} \hat{W}_{r}^{i} Q_{r}^{i}(k-1) \hat{W}_{r}^{i^{\top}}\right) \\
& =\sum_{r=1}^{m_{i}} p_{r s}^{i} \operatorname{vec}\left(\hat{W}_{r}^{i} Q_{r}^{i}(k-1) \hat{W}_{r}^{i^{\top}}\right) \\
& =\sum_{r=1}^{m_{i}} p_{r s}^{i}\left(\hat{W}_{r}^{i} \otimes \hat{W}_{r}^{i}\right) \operatorname{vec}\left(Q_{r}^{i}(k-1)\right) .
\end{aligned}
$$

In the last equality, we used the property that $\operatorname{vec}(A B C)=\left(C^{\top} \otimes A\right) \operatorname{vec}(B)$.

Now We define a new variable $y_{(\cdot)}^{i}(k) \triangleq \operatorname{vec}\left(Q_{(\cdot)}^{i}(k)\right)$, which leads to

$$
y_{s}^{i}(k)=\sum_{r=1}^{m_{i}} p_{r s}^{i}\left(\hat{W}_{r}^{i} \otimes \hat{W}_{r}^{i}\right) y_{r}^{i}(k-1) .
$$

By stacking $y_{(\cdot)}^{i}(k)$ from 1 up to $m_{i}$, with a new definition for the augmented state $\hat{y}^{i}(k) \triangleq\left[y_{1}^{i}(k)^{\top} y_{2}^{i}(k)^{\top} \ldots y_{m_{i}}^{i}(k)^{\top}\right]^{\top}$, we have the following recursion equation:

$$
\hat{y}^{i}(k)=\underbrace{\left[\begin{array}{cccc}
p_{11}^{i}\left(\hat{W}_{1}^{i} \otimes \hat{W}_{1}^{i}\right) & p_{21}^{i}\left(\hat{W}_{2}^{i} \otimes \hat{W}_{2}^{i}\right) & \ldots & p_{m_{i} 1}^{i}\left(\hat{W}_{m_{i}}^{i} \otimes \hat{W}_{m_{i}}^{i}\right) \\
p_{12}^{i}\left(\hat{W}_{1}^{i} \otimes \hat{W}_{1}^{i}\right) & p_{22}^{i}\left(\hat{W}_{2}^{i} \otimes \hat{W}_{2}^{i}\right) & \ldots & p_{m_{i} 2}^{i}\left(\hat{W}_{m_{i}}^{i} \otimes \hat{W}_{m_{i}}^{i}\right) \\
\vdots & \vdots & \ddots & \vdots \\
p_{1 m_{i}}^{i}\left(\hat{W}_{1}^{i} \otimes \hat{W}_{1}^{i}\right) & p_{2 m_{i}}^{i}\left(\hat{W}_{2}^{i} \otimes \hat{W}_{2}^{i}\right) & \ldots & p_{m_{i}}^{i}\left(\hat{W}_{m_{i}}^{i} \otimes \hat{W}_{m_{i}}^{i}\right)
\end{array}\right]}_{=\left(P^{i \top} \otimes I\right) \operatorname{diag}\left(\hat{W}_{j}^{i} \otimes \hat{W}_{j}^{i}\right)} \underbrace{\left[\begin{array}{c}
y_{1}^{i}(k-1) \\
y_{2}^{i}(k-1) \\
\vdots \\
y_{m_{i}}^{i}(k-1)
\end{array}\right]}_{=\hat{y}^{i}(k-1)} .
$$

From the above equation, it is clear that $\rho\left(\left(P^{i^{\top}} \otimes I\right) \operatorname{diag}\left(\hat{W}_{j}^{i} \otimes \hat{W}_{j}^{i}\right)\right)<$ 1 implies $\lim _{k \rightarrow \infty} \hat{y}^{i}(k)=0$, and hence this leads to $\lim _{k \rightarrow \infty} Q^{i}(k)=0 \Longleftrightarrow$ $\lim _{k \rightarrow \infty} \operatorname{tr}\left(Q^{i}(k)\right)=0 \Longleftrightarrow \lim _{k \rightarrow \infty} \mathbb{E}\left[\left\|\hat{x}_{i}(k)\right\|^{2}\right]=0$, which is the sufficient mean square stability condition for $\hat{x}_{i}(k)$. On the other hand, if we have $\rho\left(\left(P^{i^{\top}} \otimes\right.\right.$ $\left.I) \operatorname{diag}\left(\hat{W}_{j}^{i} \otimes \hat{W}_{j}^{i}\right)\right)>1$, then $\hat{y}^{i}(k)$ will diverge, resulting in necessity for the mean square stability of $\hat{x}_{i}(k)$. Hence, the spectral radius being less than one is the necessary and sufficient mean square stability condition for the state $\hat{x}_{i}(k)$. Further, we have $\lim _{k \rightarrow \infty} \mathbb{E}\left[\left\|\hat{x}_{i}(k)\right\|^{2}\right]=0, \forall i=1,2, \ldots, N \Longleftrightarrow$ $\lim _{k \rightarrow \infty} \mathbb{E}\left[\|x(k)\|^{2}\right]=0$, where $x(k)$ is the state for the DNCS defined in (5). This concludes the proof. 
Remark 4.1. Theorem 4.1 provides an efficient way to analyze the stability of large-scale DNCSs. The key idea stems from the hypothesis that the stability of each subsystem obtained by decomposing the original system will provide the stability of the entire system. Without any relaxation or conservatism, theorem 4.1 provides the necessary and sufficient condition for stability, which is equivalent to (6) developed for the mean square stability of the MJLS. Compared to the total number of modes in the full state model (5), which is $q^{N(N-1)}$, the reduced mode model ( 7$)$ has total $\sum_{i=1}^{N} q^{\hat{n}_{i}\left(\hat{n}_{i}-1\right)}$ modes. Consequently, the growth of mode numbers in full state model is exponential with respect to $N^{2}$, whereas that in reduced mode model is linear with regard to $N$. Therefore, theorem 4.1 can be checked in a computationally efficient manner.

\section{Stability Region and Stability Bound for Uncertain Markov Transition Probability Matrix}

The Markov transition probability matrix can be obtained from data of communication delays. In general, this Markov transition probability matrix obtained from statistics can be interpreted as representation of random communication delays in an average manner. Thus, one can not estimate the exact transition probability in practice, which leads to uncertainty in the Markov transition probability matrix. In this section, we aim at developing a tool to measure the stability bound for uncertain Markov transition probability matrix, in order to guarantee the stability of large-scale DNCSs with uncertain Markovian communication delays.

As explained in section 3, the dimension of the Markov transition probability matrix for the full state model is given by $P \in \mathbb{R}^{m \times m}$, where the number of switching modes is $m=q^{N(N-1)}$. For fairly large $N$, it is practically intractable to handle such a large-scale matrix. For example, even with $q=2$ and $N=100$, the mode number is given by $m=2^{100 \times 99}$, resulting in $P \in \mathbb{R}^{2^{100 \times 99} \times 2^{100 \times 99}}$. However, the reduced mode model developed in section 3 renders computation tractable because it results in huge decrease in the mode numbers as well as the dimension of each Markov transition probability matrix, by decomposing the original system into $N$ numbers of reduced mode systems. As a consequence, the reduced mode model yields $N$ numbers of new Markov transition probability matrix $P^{i}, i=1,2, \ldots, N$, which has small size. 
Here we assume that the new Markov transition probability matrix associated with the each reduced mode is given by the following form: $P^{i}=$ $\bar{P}^{i}+\Delta P^{i}, i=1,2, \ldots, N$, where $\bar{P}^{i}$ is the nominal value and $\Delta P^{i}$ is the uncertainty in the Markov transition probability matrix for $i^{\text {th }}$ subsystem. Due to the variation in $\Delta P^{i}$, the system stability may change and hence we want to estimate the bound for $\Delta P^{i}$, to guarantee the system stability. Here we assume that $\Delta P^{i}$ has the following structure:

$$
\Delta P^{i} \triangleq\left[\begin{array}{cccc}
\Delta p_{11}^{i} & \Delta p_{12}^{i} & \cdots & \Delta p_{1 m_{i}}^{i} \\
\Delta p_{21}^{i} & \Delta p_{22}^{i} & \cdots & \Delta p_{2 m_{i}}^{i} \\
\vdots & \vdots & \ddots & \vdots \\
\Delta p_{m_{i} 1}^{i} & \Delta p_{m_{i} 2}^{i} & \cdots & \Delta p_{m_{i} m_{i}}^{i}
\end{array}\right], \in \mathbb{R}^{m_{i} \times m_{i}}
$$

Since we have a constraint such that the row sum has to be zero for $\Delta P^{i}$ in above equation, we aim to find the feasible maximum bound for each row, $\varepsilon_{r}^{i}$, satisfying the inequality $\left|\Delta p_{r s}^{i}\right| \leq \varepsilon_{r}^{i}, \forall r$, in order to guarantee the system stability. Then, each $\varepsilon_{r}^{i}$ for $r=1,2, \ldots, m_{i}$ can be obtained by the following two steps.

\section{Step 1: Solve Linear Programming (LP)}

$$
\begin{array}{llll}
\text { maximize } & \mathbf{1}^{\top} z \quad \text { (for upper bound) } \\
\text { (or } \quad \begin{array}{l}
\text { minimize } \\
\mathbf{1}^{\top} z \quad(\text { for lower bound) })
\end{array} \\
\text { subject to } & \mathbb{A}|z|<b_{s}, \quad \forall s=1,2, \ldots, m_{i} \\
& l b_{s} \leq z_{s} \leq u b_{s}, \forall s=1,2, \ldots, m_{i}
\end{array}
$$

where

$$
\begin{aligned}
& z_{s} \triangleq\left[\Delta p_{1 s}^{i}, \Delta p_{2 s}^{i}, \cdots, \Delta p_{m_{i} s}^{i}\right]^{\top}, \\
& z \triangleq\left[z_{1}^{\top}, z_{2}^{\top}, \cdots, z_{m_{i}}^{\top}\right]^{\top},
\end{aligned}
$$




$$
\begin{aligned}
& \mathbb{A} \triangleq\left[\alpha_{1}, \alpha_{2}, \cdots, \alpha_{m_{i}}\right], \text { with } \alpha_{j} \triangleq\left\|\hat{W}_{j}^{i} \otimes \hat{W}_{j}^{i}\right\|_{\infty}, j=1,2, \ldots, m_{i}, \\
& b_{s} \triangleq 1-\sum_{r=1}^{m_{i}} \alpha_{r} \bar{p}_{r s}^{i}, \\
& l b_{s} \triangleq\left[-\bar{p}_{1 s}^{i},-\bar{p}_{2 s}^{i}, \cdots-\bar{p}_{m_{i} s}^{i}\right]^{\top} \\
& u b_{s} \triangleq\left[1-\bar{p}_{1 s}^{i}, 1-\bar{p}_{2 s}^{i}, \cdots 1-\bar{p}_{m_{i} s}^{i}\right]^{\top} .
\end{aligned}
$$

The inequality constraint (11) in the LP problem guarantees the mean square stability by the forthcoming Lemma 5.1 and Theorem 5.1. The term $l b_{s}$ and $u b_{s}$ in (12) are the lower and upper bounds for $z_{s}$, respectively, according to $0 \leq p_{r s}^{i}=\left(\bar{p}_{r s}^{i}+\Delta p_{r s}^{i}\right) \leq 1$.

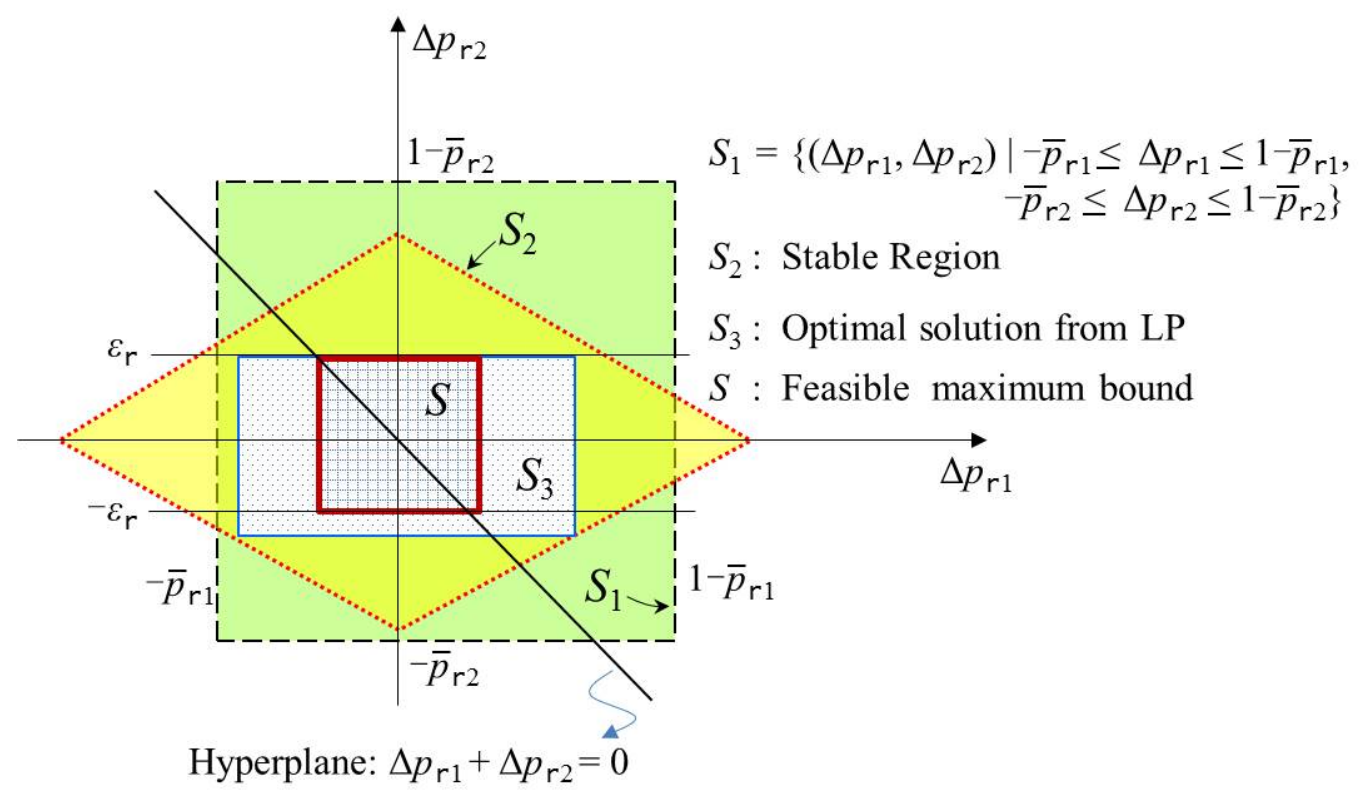

Figure 1: The geometry of the Stability Region Analysis for the uncertain Markov transition probability matrix when $m_{i}=2$. Each region is described in the figure.

\section{Step 2: Obtain Feasible Solution with Hyperplane Constraint}

We can compute the feasible maximum bound for $\Delta p_{r s}^{i}$ as follows.

$$
\varepsilon_{r}^{i}=\min \left(\min \left(\left|\varepsilon_{r, \mathrm{lb}}^{i}\right|\right), \min \left(\left|\varepsilon_{r, \mathrm{ub}}^{i}\right|\right)\right), \quad r=1,2, \ldots, m_{i} .
$$


where $\varepsilon_{r, \mathrm{lb}}^{i} \triangleq\left[\left(\Delta p_{r 1}^{i}\right)_{\mathrm{lb}}^{\star},\left(\Delta p_{r 2}^{i}\right)_{\mathrm{lb}}^{\star}, \ldots,\left(\Delta p_{r m}^{i}\right)_{\mathrm{lb}}^{\star}\right]^{\top}, \varepsilon_{r, \mathrm{ub}}^{i} \triangleq\left[\left(\Delta p_{r 1}^{i}\right)_{\mathrm{ub}}^{\star},\left(\Delta p_{r 2}^{i}\right)_{\mathrm{ub}}^{\star}\right.$, $\left.\ldots,\left(\Delta p_{r m}^{i}\right)_{\mathrm{ub}}^{\star}\right]^{\top}$, and $\left(\Delta p_{r s}^{i}\right)_{l b}^{\star},\left(\Delta p_{r s}^{i}\right)_{u b}^{\star}$ denote optimal lower and upper bounds for $\Delta p_{r s}^{i}$, obtained from the LP, respectively.

Since upper or lower bounds are solved by maximizing or minimizing the objective function, $\left(\Delta p_{r s}^{i}\right)^{\star}$ has different values for upper and lower bounds. Fig.1 shows the geometry of stability region analysis for uncertain transition probability matrix. The region $S_{1}$ stands for the bounds that come from $-\bar{p}_{r s}^{i} \leq \Delta p_{r s}^{i} \leq 1-\bar{p}_{r s}^{i}$. $\quad S_{2}$ can be obtained from inequality constraint (11). The region $S_{3}$ denotes the solution from the LP, and $S$ is the feasible maximum bound with a stability guarantee. Note that $\Delta P^{i}$ satisfies $\sum_{s=1}^{m_{i}} \Delta p_{r s}^{i}=0, \forall r$ and hence, feasible solutions should lie on the hyperplane, satisfying $\Delta p_{r 1}^{i}+\Delta p_{r 2}^{i}+\ldots+\Delta p_{r m_{i}}^{i}=0, \forall r$. Therefore, we can compute the feasible maximum bound from (13) for each row $r$.

Now we prove that the inequality constraint (11) guarantees the system stability. For this purpose, the following Lemma 5.1 will be used.

Lemma 5.1. Consider a block matrix $X$ defined by

$$
X=\left[\begin{array}{cccc}
X_{11} & X_{12} & \cdots & X_{1 m} \\
X_{21} & X_{22} & \cdots & X_{2 m} \\
\vdots & \vdots & \vdots & \vdots \\
X_{m 1} & X_{m 2} & \cdots & X_{m m}
\end{array}\right]
$$

where matrix $X_{i j} \in \mathbb{R}^{n \times n}$. Then, we have $\rho(X)<1$, if $\sum_{j=1}^{m}\left\|X_{i j}\right\|_{\infty}<1, \forall i=$ $1,2, \ldots, m$.

Proof. For the block matrix $X$ given above, the following inequality condition $\|X\|_{\infty} \leq \max _{i} \sum_{j=1}^{m}\left\|X_{i j}\right\|_{\infty}$ holds. Also, it is well known that $\rho(X) \leq$ $\|X\|_{p}$ for any choice of $p$.

Therefore, we conclude that $\sum_{j=1}^{m}\left\|X_{i j}\right\|_{\infty}<1, \forall i=1,2, \ldots, m \Rightarrow$ $\rho(X) \leq\|X\|_{\infty}<1$

Theorem 5.1. Consider the MJLS (5) for the large-scale DNCS with communication delays. Then, (5) is mean square stable if

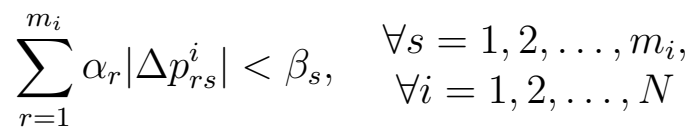


where $\alpha_{r}=\left\|\hat{W}_{r}^{i} \otimes \hat{W}_{r}^{i}\right\|_{\infty}$ and $\beta_{s}=1-\sum_{r=1}^{m_{i}} \bar{p}_{r s}^{i}\left\|\hat{W}_{r}^{i} \otimes \hat{W}_{r}^{i}\right\|_{\infty}$, is satisfied.

PROOF. If the Markov transition probability matrix for the system in (7) has the uncertainty denoted by $P^{i}=\bar{P}^{i}+\Delta P^{i}$, then the term $\rho\left(\left(P^{i^{\top}} \otimes I\right)\right.$ $\left.\operatorname{diag}\left(\hat{W}_{j}^{i} \otimes \hat{W}_{j}^{i}\right)\right)$ in (8) can be expressed as

$$
\begin{aligned}
& \rho\left(\left(P^{i^{\top}} \otimes I\right) \operatorname{diag}\left(\hat{W}_{j}^{i} \otimes \hat{W}_{j}^{i}\right)\right) \\
= & \rho\left(\left(\left(\bar{P}^{i}+\Delta P^{i}\right)^{\top} \otimes I\right) \operatorname{diag}\left(\hat{W}_{j}^{i} \otimes \hat{W}_{j}^{i}\right)\right) \\
= & \rho\left(\left(\left(\bar{P}^{i^{\top}} \otimes I\right)+\left(\Delta P^{i^{\top}} \otimes I\right)\right) \operatorname{diag}\left(\hat{W}_{j}^{i} \otimes \hat{W}_{j}^{i}\right)\right) \\
= & \rho\left(\left(\bar{P}^{i^{\top}} \otimes I\right) \operatorname{diag}\left(\hat{W}_{j}^{i} \otimes \hat{W}_{j}^{i}\right)+\left(\Delta P^{i^{\top}} \otimes I\right) \operatorname{diag}\left(\hat{W}_{j}^{i} \otimes \hat{W}_{j}^{i}\right)\right) \\
\leq & \left\|\left(\bar{P}^{i}{ }^{\top} \otimes I\right) \operatorname{diag}\left(\hat{W}_{j}^{i} \otimes \hat{W}_{j}^{i}\right)+\left(\Delta P^{i^{\top}} \otimes I\right) \operatorname{diag}\left(\hat{W}_{j}^{i} \otimes \hat{W}_{j}^{i}\right)\right\|_{\infty} \\
\leq & \left\|\left(\bar{P}^{i^{\top}} \otimes I\right) \operatorname{diag}\left(\hat{W}_{j}^{i} \otimes \hat{W}_{j}^{i}\right)\right\|_{\infty}+\left\|\left(\Delta P^{i^{\top}} \otimes I\right) \operatorname{diag}\left(\hat{W}_{j}^{i} \otimes \hat{W}_{j}^{i}\right)\right\|_{\infty},
\end{aligned}
$$

In the first inequality, we used the fact that $\rho(\cdot) \leq\|\cdot\|_{\infty}$ and the submultiplicative property was applied in the last inequality. The block matrix structure for each term of the last inequality is alternatively expressed as follows:

$$
\begin{aligned}
& \left\|\left(\bar{P}^{i}{ }^{\top} \otimes I\right) \operatorname{diag}\left(\hat{W}_{j}^{i} \otimes \hat{W}_{j}^{i}\right)\right\|_{\infty}
\end{aligned}
$$

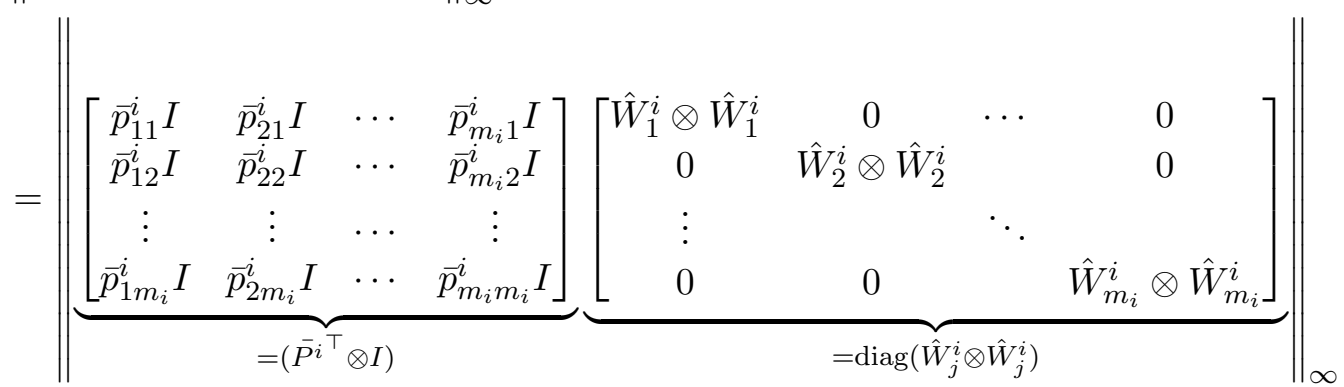

$$
\begin{aligned}
& =\left\|\begin{array}{cccc}
\gamma_{1} \bar{p}_{11}^{i} & \gamma_{2} \bar{p}_{21}^{i} & \cdots & \gamma_{m_{i}} \bar{p}_{m_{i} 1}^{i} \\
\gamma_{1} \bar{p}_{12}^{i} & \gamma_{2} \bar{p}_{22}^{i} & \cdots & \gamma_{m_{i}} \bar{p}_{m_{i} 2}^{i} \\
\vdots & \vdots & \ddots & \vdots \\
\gamma_{1} \bar{p}_{1 m_{i}}^{i} & \gamma_{2} \bar{p}_{2 m_{i}}^{i} & \cdots & \gamma_{m_{i}} \bar{p}_{m_{i} m_{i}}^{i}
\end{array}\right\|_{\infty},
\end{aligned}
$$


where $\gamma_{j}=\left(\hat{W}_{j}^{i} \otimes \hat{W}_{j}^{i}\right), j=1,2, \ldots, m_{i}$, and similarly,

$$
\left\|\left(\Delta P^{i^{\top}} \otimes I\right) \operatorname{diag}\left(\hat{W}_{j}^{i} \otimes \hat{W}_{j}^{i}\right)\right\|_{\infty}=\left\|\begin{array}{cccc}
\gamma_{1} \Delta p_{11}^{i} & \gamma_{2} \Delta p_{21}^{i} & \cdots & \gamma_{m_{i}} \Delta p_{m_{i} 1}^{i} \\
\gamma_{1} \Delta p_{12}^{i} & \gamma_{2} \Delta p_{22}^{i} & \cdots & \gamma_{m_{i}} \Delta p_{m_{i} 2}^{i} \\
\vdots & \vdots & \ddots & \vdots \\
\gamma_{1} \Delta p_{1 m_{i}}^{i} & \gamma_{2} \Delta p_{2 m_{i}}^{i} & \cdots & \gamma_{m_{i}} \Delta p_{m_{i} m_{i}}^{i}
\end{array}\right\|_{\infty}
$$

By applying the result in Lemma 5.1 into (14), it is guaranteed that $\rho\left(\left(P^{i^{\top}} \otimes I\right) \operatorname{diag}\left(\hat{W}_{j}^{i} \otimes \hat{W}_{j}^{i}\right)\right)<1$, if the following condition

$$
\sum_{r=1}^{m_{i}} \alpha_{r}\left|\Delta p_{r s}^{i}\right|+\sum_{r=1}^{m_{i}} \alpha_{r} \bar{p}_{r s}^{i}<1, \quad \forall s=1,2, \ldots, m_{i}
$$

where $\alpha_{r} \triangleq\left\|\hat{W}_{r}^{i} \otimes \hat{W}_{r}^{i}\right\|_{\infty}$, is satisfied.

Therefore, (5) is mean square stable by Theorem 4.1 if it is guaranteed that

$$
\sum_{r=1}^{m_{i}} \alpha_{r}\left|\Delta p_{r s}^{i}\right|<\beta_{s}, \quad \begin{aligned}
& \forall s=1,2, \ldots, m_{i} \\
& \forall i=1,2, \ldots, N
\end{aligned}
$$

where $\beta_{s} \triangleq 1-\sum_{r=1}^{m_{i}} \alpha_{r} \bar{p}_{r s}^{i}$

\section{Numerical Example}

We consider the DNCS that has random communication delays, where the total number of agents is denoted by $N$. The state for each agent is updated by the following scalar discrete-time dynamics:

$$
\begin{aligned}
& x_{i}(k+1)=\sum_{j \in \mathcal{N}_{i}} a_{i j} x_{j}\left(k^{*}\right), \quad i=1,2, \ldots, N, \\
& \text { where } a_{i j} \triangleq \begin{cases}0.7, & j=i, \\
-0.14, & j \in\{i-1, i+1\}, \\
0, & \text { otherwise. }\end{cases}
\end{aligned}
$$


When there is no communication delay (i.e., $k^{*}=k$ ), the dynamics for the entire DNCS is obtained by (2) as follows:

$$
\mathcal{A}=\left[\begin{array}{cccccc}
0.7 & -0.14 & 0 & 0 & \cdots & 0 \\
-0.14 & 0.7 & -0.14 & 0 & \cdots & 0 \\
0 & -0.14 & 0.7 & -0.14 & \cdot & 0 \\
\vdots & \cdot & \ddots & \ddots & \ddots & \vdots \\
0 & \cdot & \cdot & -0.14 & 0.7 & -0.14 \\
0 & 0 & \ldots & 0 & -0.14 & 0.7
\end{array}\right] \in \mathbb{R}^{N \times N}
$$

In this case, the stability is computed by $\rho(\mathcal{A})=0.9523<1$, which assures that the given DNCS without communication delays is stable. However, communications (e.g., wireless network) may be delayed in practice and thus, above stability result is no longer guaranteed. Therefore, it is required to test the system stability in the existence of random communication delays.

\subsection{Stability Analysis with random communication delays}

We assume that the communication delay $\tau$ is bounded by $0 \leq \tau \leq \tau_{d}=1$, i.e., $k^{*}=\{k, k-1\}, \forall i=1,2, \ldots, N$, which implies $q=2$. Also, we assume that all communication delays for each agent (15) are governed by the Markov process with an initial probability distribution $\pi(0)$ and the Markov transition probability matrix $P$, given by

$$
\pi(0)=[1,0], \quad P=\left[\begin{array}{ll}
0.6 & 0.4 \\
0.3 & 0.7
\end{array}\right] .
$$

For this system, even with $N=100$, the full state model (5) has total $q^{N(N-1)}=2^{100 \times 99}$ modes. It is computationally intractable to deal with $2^{100 \times 99}$ numbers of subsystem dynamics, in order to analyze system stability. In contrast, the reduce mode model (7) has total $\sum_{i=1}^{N} q^{\hat{n}_{i}\left(\hat{n}_{i}-1\right)}=98 \times$ $\left(2^{3 \times 2}\right)+2 \times\left(2^{2 \times 1}\right)=6280$ modes. Furthermore, the proposed method to reduce the mode numbers fully maximizes its own advantage by considering the symmetric property between agents, which cannot be implemented on the full state model. Since subsystems are symmetric for $\forall i=2,3, \ldots, N-1$ and for $\forall i=1, N$, we only need to check the stability condition for these two cases. Taking into account the symmetric structure, the reduced mode model results in total $2^{3 \times 2}+2^{2 \times 1}=68$ modes. Compared to $2^{100 \times 99}$ numbers of modes, the proposed method leads to huge decrease in the mode numbers. 


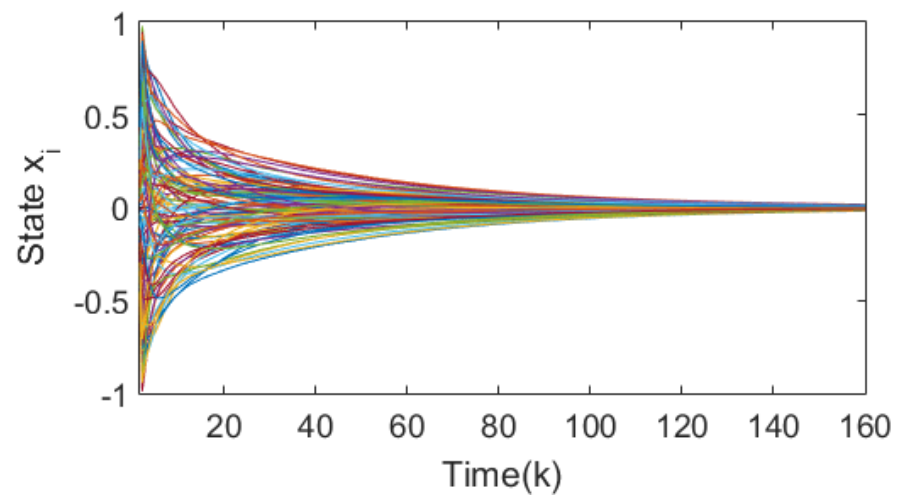

Figure 2: State trajectories of each agent for the DNCS with the Markovian communication delays. Initial conditions are randomly generated for the state $x_{i}, i=1,2, \ldots, 100$.

The spectral radius for $i=2,3, \ldots, 99$ is computed by $\rho\left(\left(P^{i^{\top}} \otimes I\right)\right.$ $\left.\operatorname{diag}\left(\hat{W}_{j}^{i} \otimes \hat{W}_{j}^{i}\right)\right)=0.8208<1$, where $P^{i}=(P \otimes P \otimes P \otimes P)$. For $i=1$ and $N$, we have $\rho\left(\left(P^{i^{\top}} \otimes I\right) \operatorname{diag}\left(\hat{W}_{j}^{i} \otimes \hat{W}_{j}^{i}\right)\right)=0.7214<1$, where $P^{i}=(P \otimes P)$. Consequently, this system is stable in the mean square sense by Theorem 4.1. The state trajectory plot also supports this result, as shown in Fig. 2. For this simulation, initial condition was assumed to be uniformly distributed in $[-1,1]$, and was generated by manipulating the MATLAB ${ }^{\circledR}$ command rand $(\ldots)$ that generates uniformly distributed pseudo random numbers between 0 and 1 .

\subsection{Stability Bound for uncertain Markov transition probability matrix}

In order to solve the LP to estimate the bound for uncertain Markov transition probability matrix, we used MATLAB ${ }^{\circledR}$ with CVX[24], a Matlabbased software for convex optimization.

\subsubsection{Scalar system}

Although the proposed method to estimate maximum bound for uncertain Markov transition probability matrix is developed for the large-scale DNCS, it is also applicable to general MJLS. We adopted a following example, introduced in [23] to compare the performance of maximum bound estimation. 
Consider the following MJLS that has two modes with scalar discrete-time dynamics.

$$
\begin{aligned}
& x(k+1)=a_{\sigma(k)} x(k), \quad \sigma(k) \in\{1,2\} \\
& a_{1}=1 / 2, \quad a_{2}=5 / 4 .
\end{aligned}
$$

The Markov transition probability matrix has the form of $P=\bar{P}+\Delta P$, where

$$
\bar{P}=\left[\begin{array}{ll}
0.4 & 0.6 \\
0.5 & 0.5
\end{array}\right], \Delta P=\left[\begin{array}{ll}
\Delta p_{11} & \Delta p_{12} \\
\Delta p_{21} & \Delta p_{22}
\end{array}\right], \sum_{j=1}^{2} \Delta p_{i j}=0, \quad \forall i=1,2
$$

After applying the two steps proposed in this paper, we obtained the maximum bound $\varepsilon_{1}=0.4, \varepsilon_{2}=0.02$ whereas [23] gives the value as $\varepsilon_{1}=\varepsilon_{2}=$ 0.021, which is more conservative. For stability check, among all possible scenarios with $\left|\Delta p_{r s}\right| \leq \varepsilon_{r}, \forall r, s=1,2$, we have $\max \rho\left(\left(P^{\top} \otimes I\right) \operatorname{diag}\left(a_{j} \otimes\right.\right.$ $\left.\left.a_{j}\right)\right)=1$, which is a marginal value for stability. Hence, the system is stable with obtained maximum bound that is more relaxed than [23].

\subsubsection{The DNCS with random communication delays}

Recalling the DNCS example, let us denote $\bar{P}$ as a nominal Markov transition probability matrix, which represents the transition probability in $k^{*} \in\{k, k-1\}$. We assume that $\bar{P}$ has the same structure with $P$ in (16). Then, the new Markov transition probability matrix associated with the reduced mode model is calculated by $\bar{P}^{i}=\bar{P} \otimes \bar{P}$ for $i=1, N$ and $\bar{P}^{i}=(\bar{P} \otimes \bar{P} \otimes \bar{P} \otimes \bar{P})$ for $i=2,3, \ldots, N-1$. More details on synthesizing new Markov transition probability matrix can be found in [6] (see Page 2001).

The feasible solution with the LP provides the maximum bound $\varepsilon^{i}=$ $\left[\varepsilon_{1}^{i}, \varepsilon_{2}^{i}, \ldots \varepsilon_{16}^{i}\right]=10^{-2} \times[0.37,0.23,0.23,0.86,0.23,0.86,0.86,1.23,0.23$, $0.86,0.86,1.23,0.86,1.23,1.23,1.72], \forall i=2,3, \ldots, N-1$. For $i=1$ and $N$, we obtained $\varepsilon^{i}=\left[\varepsilon_{1}^{i}, \varepsilon_{2}^{i}, \varepsilon_{3}^{i}, \varepsilon_{4}^{i}\right]=10^{-2} \times[0.75,2.25,2.25,3.75]$. Therefore, we can assure that $N$ inverted pendulum system is mean square stable if the uncertainty in the Markov transition probability matrix is within the bound such that $\left|\Delta p_{r s}^{i}\right| \leq \varepsilon_{r}^{i}, \forall r$ and $\forall i=1,2, \ldots, N$. 


\section{Conclusions}

This paper studied the mean square stability of large-scale DNCSs in connection with random communication delays. Although the Markov jump linear system has been widely adopted to model the systems associated with random communication delays, it inevitably results in a huge number of modes for the large-scale DNCSs, making current stability analysis tools intractable. To avoid this scalability problem, we provided a new analysis framework, which incorporates a reduced mode model that scales linearly with respect to the number of agents. Additionally, we presented a new method to estimate stability bound for uncertain Markov transition probability matrix, in order to guarantee the stability of DNCSs. We showed that this method is less conservative than those proposed in the literature. The validity of the proposed methods were verified by the numerical example.

\section{Acknowledgements}

This research was supported by the National Science Foundation award \#1349100.

[1] Yuan-Chieh Cheng and Thomas G Robertazzi. Distributed computation with communication delay (distributed intelligent sensor networks). Aerospace and Electronic Systems, IEEE Transactions on, 24(6):700-712, 1988.

[2] Gregory C Walsh, Hong Ye, and Linda G Bushnell. Stability analysis of networked control systems. Control Systems Technology, IEEE Transactions on, 10(3):438-446, 2002.

[3] John K Yook, Dawn M Tilbury, and Nandit R Soparkar. Trading computation for bandwidth: Reducing communication in distributed control systems using state estimators. Control Systems Technology, IEEE Transactions on, 10(4):503-518, 2002.

[4] Fuwen Yang, Zidong Wang, YS Hung, and Mahbub Gani. H control for networked systems with random communication delays. Automatic Control, IEEE Transactions on, 51(3):511-518, 2006.

[5] Johan Nilsson, Bo Bernhardsson, and Björn Wittenmark. Stochastic analysis and control of real-time systems with random time delays. Automatica, 34(1):57-64, 1998.

[6] Lin Xiao, Arash Hassibi, and Jonathan P How. Control with random communication delays via a discrete-time jump system approach. In American Control Conference, 2000. Proceedings of the 2000, volume 3, pages 2199-2204. IEEE, 2000. 
[7] Kooktae Lee, Abhishek Halder, and Raktim Bhattacharya. Performance and robustness analysis of stochastic jump linear systems using wasserstein metric. Automatica, 51:341-347, 2015.

[8] Kooktae Lee, Abhishek Halder, and Raktim Bhattacharya. Probabilistic robustness analysis for stochastic jump linear systems. In American Control Conference (ACC), 2014. Proceedings of the 2014, pages 2638-2643. IEEE, 2014.

[9] Xudong Zhao, Shen Yin, Hongyi Li, and Ben Niu. Switching stabilization for a class of slowly switched systems. Automatic Control, IEEE Transactions on, 60(1):221-226, 2014.

[10] Xiangbo Feng, Kenneth A Loparo, Yuandong Ji, and Howard Jay Chizeck. Stochastic stability properties of jump linear systems. Automatic Control, IEEE Transactions on, 37(1):38-53, 1992.

[11] Yuguang Fang and Kenneth A Loparo. Stabilization of continuous-time jump linear systems. Automatic Control, IEEE Transactions on, 47(10):1590-1603, 2002.

[12] Peng Shi, E-K Boukas, and Ramesh K Agarwal. Control of markovian jump discretetime systems with norm bounded uncertainty and unknown delay. Automatic Control, IEEE Transactions on, 44(11):2139-2144, 1999.

[13] Pete Seiler and Raja Sengupta. Analysis of communication losses in vehicle control problems. In American Control Conference, 2001. Proceedings of the 2001, volume 2, pages 1491-1496. IEEE, 2001.

[14] Liqian Zhang, Yang Shi, Tongwen Chen, and Biao Huang. A new method for stabilization of networked control systems with random delays. Automatic Control, IEEE Transactions on, 50(8):1177-1181, 2005.

[15] Yang Shi and Bo Yu. Output feedback stabilization of networked control systems with random delays modeled by markov chains. Automatic Control, IEEE Transactions on, 54(7):1668-1674, 2009.

[16] Ming Liu, Daniel WC Ho, and Yugang Niu. Stabilization of markovian jump linear system over networks with random communication delay. Automatica, 45(2):416-421, 2009.

[17] David Sworder. Feedback control of a class of linear systems with jump parameters. Automatic Control, IEEE Transactions on, 14(1):9-14, 1969.

[18] Oswaldo LV Costa and Marcelo D Fragoso. Stability results for discrete-time linear systems with markovian jumping parameters. Journal of mathematical analysis and applications, 179(1):154-178, 1993.

[19] Oswaldo Luiz do Valle Costa, Marcelo Dutra Fragoso, and Ricardo Paulino Marques. Discrete-time Markov jump linear systems. Springer, 2006. 
[20] Lixian Zhang and El-Kébir Boukas. Stability and stabilization of markovian jump linear systems with partly unknown transition probabilities. Automatica, 45(2):463468, 2009.

[21] Kooktae Lee, Raktim Bhattacharya, and Vijay Gupta. A switched dynamical system framework for analysis of massively parallel asynchronous numerical algorithm. In American Control Conference (ACC), 2015. Proceedings of the 2015, pages 10951100. IEEE, 2015.

[22] Lixian Zhang and James Lam. Necessary and sufficient conditions for analysis and synthesis of markov jump linear systems with incomplete transition descriptions. Automatic Control, IEEE Transactions on, 55(7):1695-1701, 2010.

[23] Mehmet Karan, Peng Shi, and C Yalçın Kaya. Transition probability bounds for the stochastic stability robustness of continuous-and discrete-time markovian jump linear systems. Automatica, 42(12):2159-2168, 2006.

[24] Michael Grant, Stephen Boyd, and Yinyu Ye. Cvx: Matlab software for disciplined convex programming, 2008. 